\title{
On the Model-Based Control of Networked Systems
}

\author{
Luis A. Montestruque, Panos J. Antsaklis \\ University of Notre Dame, Notre Dame, IN 46556, U.S.A. \\ Department of Electrical Engineering \\ fax: (574) 631-4393 \\ e-mail: \{lmontest, pantsakl\}@nd.edu
}

\begin{abstract}
In this paper the control of linear plants, where the sensor is connected to a linear controller/actuator via a network is addressed. Both, state and output feedback, are considered and results are derived for both continuous and discrete plants. A key idea is that knowledge of the plant dynamics is used to reduce the usage of the network. Necessary and sufficient conditions for stability are derived as simple eigenvalue tests of a well-structured test matrix, constructed in terms of the update time $h$, and the parameters of the plant and of its model. These tests are extended to include network delay as well.
\end{abstract}

Key words: Networked Control Systems, Stabilizability, Hybrid Systems, Minimum Feedback Information.

\section{Introduction}

The use of networks as a media to interconnect the different components in an industrial control system is rapidly increasing. A network introduces bandwidth restrictions. To overcome these bandwidth constraints several approaches have been proposed. Some of these include the study of networked systems with transport delays (Matveev \& Savkin, 2001), and under noise disturbances (Petersen \& Savkin, 2001), quantization effects and algorithms (Elia \& Mitter, 2001; Ishii \& Francis, 2000; Nair \& Evans, 2000), and scheduling algorithms (HristuVarsakelis, 2001; Rehbinder \& Sanfridson, 2000; Matveev \& Savkin, 2001; Walsh, Beldiman, \& Bushnell, 1999).

It is clear that the reduction of bandwidth necessitated by the communication network in a networked control system is a major concern. In this paper we explore the effect of reducing the number of data packet exchanges between the sensor and the controller/actuator. In particular, we identify the maximum allowed transfer time between the sensor and the actuator for the system to be stable. In order to increase the transfer time we will use the intuitive idea of using the knowledge we have of the plant dynamics as it is contained in the plant model. The plant model is used at the controller/actuator side to approximate the plant behavior during time periods when sensor data are not available. The main idea is to perform the feedback by updating the model's state using the actual state of the plant that is provided by the sensor. The rest of the time the control action is based on a plant model that is incorporated in the controller/actuator and is running open loop for a period of $h$ seconds (or samples, in the case of discrete plants). The setup for a continuous plant is shown in Figure1. Output feedback on a continuous plant is used in Figure 2.
This idea of a tradeoff between open loop and closed loop control is related to the minimal attention control proposed by Brockett (1997). One of the main differences resides in that minimal attention control makes this tradeoff in a continuous way while the proposed setup does this tradeoff in a "discrete" manner.

The compensated plant model with its update scheme on Figure 1 can also be seen as a type of hold system. Mirkin, Rotstein, \& Palmor (1999) explore the joint optimization of a generalized hold, sampler, and controller for a sampled system using a technique known as lifting. It turns out that our model resembles the $\mathrm{H}_{2}$ optimal hold. However, these results don't explicitly incorporate the plant-model error, the discrete plant case, or the inclusion of transport delays on the network.

Our approach incorporates a model of the plant, the state of which is updated with the plant's state. We derive a necessary and sufficient condition for stability that results in a maximum transfer time, which depends on the model inaccuracies. In the absence of plant disturbances arbitrarily long transfer times can be achieved depending on modeling errors.

For our analysis we will assume that the compensated model is stable and that the transportation delay is negligible. We will assume that the frequency at which the network updates the state in the controller is constant. The idea is to find the smallest frequency at which the network must update the state in the controller, that is, an upper bound for $h$, the update time. Usual assumptions in the literature include requiring a stable plant or a smaller update time than the sampling time in the case of a discrete controller. Here we do not make any of these assumptions. The plant may be unstable. These results are extended to the output feedback case as well. 


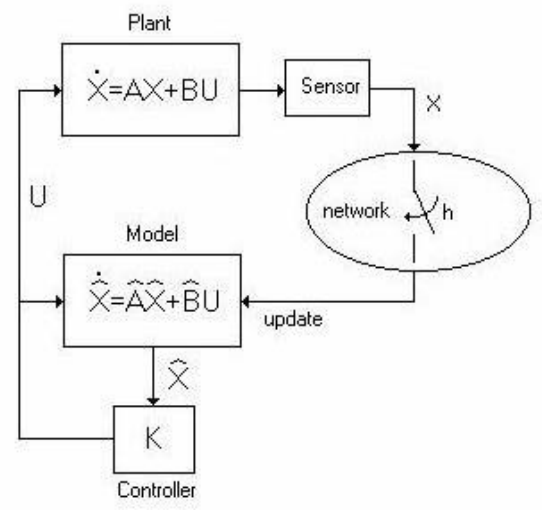

Figure 1: Proposed configuration of a state feedback networked control system.

This paper is organized as follows: in Section 2 necessary and sufficient conditions are developed for the control architecture shown in Figure 1. This is the case where the state vector is directly measurable and sent through the network to the controller/actuator. The case of output feedback control is analyzed in Section 3. An extension to discrete systems is given in Section 4. An extension of the state feedback system in the presence of network delays is presented in Section 5. A numeric example is given in Appendix A. Conclusions are presented in Section 6.

\section{A State Feedback Networked Control System}

Consider the control system of Figure 1 where the actual plant behavior is described by $\dot{x}=A x+B u$, the plant model is given by $\dot{\hat{x}}=\hat{A} \hat{x}+\hat{B} u$, and the controller by $u=K \hat{x}$.

Since the sensor has the full state vector available, its function will be to send the state information through the network every $h$ seconds. The state error is defined as $e=x-\hat{x}$, and represents the difference between the plant state and the model state. The modeling error matrices $\widetilde{A}=A-\hat{A}$ and $\widetilde{B}=B-\hat{B}$ represent the difference between the plant and the model. Finally, the update times are $t_{k}$, where $t_{k}-t_{k-1}=h$ for all $k$. Since the model state is updated every $t_{k}$ seconds, $e\left(t_{k}\right)=0$ for $k=0,1,2, \ldots$. This resetting of the state error every update time is key of our control system. It is easy to see that the dynamics of the overall system for $t \in\left[t_{k}, t_{k+1}\right)$ can be described by

$$
\dot{z}=\Lambda z \text { for } t \in\left[t_{k}, t_{k+1}\right) \text { and } z\left(t_{k}\right)=\left[\begin{array}{ll}
x\left(t_{k}^{-}\right) & 0
\end{array}\right]^{T}
$$

where $z(t)=\left[\begin{array}{ll}x(t) & e(t)\end{array}\right]^{T}$, and $\Lambda=\left[\begin{array}{cc}A+B K & -B K \\ \tilde{A}+\tilde{B} K & \hat{A}-\tilde{B} K\end{array}\right]$.

\section{Proposition \#1}

The system described by Equation (1) with initial conditions $z\left(t_{0}\right)=\left[\begin{array}{lll}x\left(t_{0}\right) & 0\end{array}\right]^{T}=z_{0}$, has the following response:

$$
\begin{aligned}
& z(t)=e^{\Lambda\left(t-t_{k}\right)}\left(\left[\begin{array}{ll}
I & 0 \\
0 & 0
\end{array}\right] e^{\Lambda h}\left[\begin{array}{ll}
I & 0 \\
0 & 0
\end{array}\right]\right)^{k} z_{0}, \\
& \text { for } t \in\left[t_{k}, t_{k+1}\right), \text { with } t_{k+1}-t_{k}=h
\end{aligned}
$$

Proof.

On the interval $t \in\left[t_{k}, t_{k+1}\right)$, the system response is

$$
z(t)=\left[\begin{array}{l}
x(t) \\
e(t)
\end{array}\right]=e^{\Lambda\left(t-t_{k}\right)}\left[\begin{array}{l}
x\left(t_{k}\right) \\
0
\end{array}\right]=e^{\Lambda\left(t-t_{k}\right)} z\left(t_{k}\right)
$$

Now, note that at times $t_{k}, z\left(t_{k}\right)=\left[\begin{array}{lll}x\left(t_{k}^{-}\right) & 0\end{array}\right]^{T}$, that is, the error $e(t)$ is reset to zero. We can represent this by $z\left(t_{k}\right)=I_{S^{z}}\left(t_{k}^{-}\right)$where $I_{S}=\left[\begin{array}{ll}I & 0 \\ 0 & 0\end{array}\right]$. Using Equation

to calculate $z\left(t_{k}^{-}\right)$we obtain: $z\left(t_{k}\right)=I_{S} e^{\Lambda h} z\left(t_{k-1}\right)$.

In view of (3) we have that if at time $t=t_{0}$, $z\left(t_{0}\right)=z_{0}=\left[\begin{array}{ll}x_{0} & 0\end{array}\right]^{T}$ is the initial condition then

$$
z(t)=e^{\Lambda\left(t-t_{k}\right)}\left(I_{S} e^{\Lambda h}\right)^{k} z_{0}=e^{\Lambda\left(t-t_{k}\right)}\left(I_{S} e^{\Lambda h} I_{S}\right)^{k} z_{0}
$$

We now state the following theorem characterizing the necessary and sufficient conditions for the system described by Equation (1) to have global exponential stability around the solution $z=0$. The norm used here is the 2norm but any other consistent norm can also be used.

\section{Theorem \#1}

The system described by Equation (1) is globally exponentially stable around the solution $z=\left[\begin{array}{ll}x & e\end{array}\right]^{T}=\left[\begin{array}{ll}0 & 0\end{array}\right]^{T}$ if and only if the eigenvalues of $M=\left[\begin{array}{ll}I & 0 \\ 0 & 0\end{array}\right] e^{\Lambda h}\left[\begin{array}{ll}I & 0 \\ 0 & 0\end{array}\right]$ are strictly inside the unit circle.

\section{Proof.}

Sufficiency: Taking the norm of the solution described as in Proposition 1:

$$
\|z(t)\|=\left\|e^{\Lambda\left(t-t_{k}\right)} M^{k} z_{0}\right\| \leq\left\|e^{\Lambda\left(t-t_{k}\right)}\right\| \cdot\left\|M^{k}\right\| \cdot\left\|z_{0}\right\|
$$

Analyzing the first term on the right hand side of (5):

$$
\left\|e^{\Lambda\left(t-t_{k}\right)}\right\| \leq e^{\sigma(\Lambda)\left(t-t_{k}\right)} \leq e^{\sigma(\Lambda) h}=K_{1}
$$

where $\bar{\sigma}(\Lambda)$ is the largest singular value of $\Lambda$. In general this term can always be bounded since the time difference $t-t_{k}$ is always smaller than $h$.

We now study the term $\left\|M^{k}\right\|$. It is clear that this term will be bounded if and only if the eigenvalues of $M$ lie inside the unit circle $\left\|M^{k}\right\| \leq K_{2} e^{-\alpha_{1} k} \leq K_{3} e^{-\alpha t}$ with $K_{2}, K_{3}, \alpha_{1}, \alpha>0$. So we can conclude that $\|z(t)\| \leq K_{1} \cdot K_{3} e^{-\alpha t} \cdot\left\|z_{0}\right\|$.

Necessity: We will now prove the necessity part of the theorem by contradiction. Assume the system described by (1) is stable and that $M$ has at least one eigenvalue outside the unit circle. Since the system is stable, a periodic sample 
of the response should be stable as well. We will take the sample at times $t_{k+1}^{-}$, that is, just before the update. We will concentrate on a specific term: the state of the plant $x\left(t_{k+1}^{-}\right)$, which is the first element of $z\left(t_{k+1}^{-}\right)$. We will call $x\left(t_{k+1}^{-}\right), \xi(k)$. Now assume $e^{\Lambda \tau}$ has the following form: $e^{\Lambda \tau}=\left[\begin{array}{ll}W(\tau) & X(\tau) \\ Y(\tau) & Z(\tau)\end{array}\right]$. Now the values of the solution at times $t_{k+1}^{-}$, that is just before the update, are

$z\left(t_{k+1}^{-}\right)=\left[\begin{array}{cc}W(h)(W(h))^{k} & 0 \\ Y(h)(W(h))^{k} & 0\end{array}\right] z_{0}=\left[\begin{array}{cc}(W(h))^{k+1} & 0 \\ Y(h)(W(h))^{k} & 0\end{array}\right] z_{0}$

It is clear that, since $M$ has at least one eigenvalue outside the unit circle (and thus in $W$ ), $\xi(k)$ will in general grow with $k$. This clearly means the system cannot be stable, and thus we have a contradiction.

\section{Discussion.}

One can gain insight into the closed loop system behavior by noticing that the nonzero eigenvalues of the test matrix $M$ are exactly the eigenvalues of another matrix:

$$
N=e^{(\hat{A}+\hat{B} K) h}+e^{A h} \int_{0}^{h} e^{-A \tau}(\tilde{A}+\tilde{B} K) e^{(\hat{A}+\hat{B} K) \tau} d \tau
$$

This can be shown either directly or by using Laplace transform (see Montestruque \& Antsaklis, 2002).

Note that the eigenvalues of the compensated model appear in the first term of $N$. We can view the term $\Delta=e^{A h} \int_{0}^{h} e^{-A \tau}(\tilde{A}+\tilde{B} K) e^{(\hat{A}+\hat{B} K) \tau} d \tau$ as a perturbation on the desired eigenvalues, that is, the eigenvalues of the compensated model. Even if the eigenvalues of the original plant were unstable the perturbation $\Delta$ can be made small enough by having $h$ and $\tilde{A}+\tilde{B} K$ small and thus minimizing their impact over the eigenvalues of the compensated plant. We also observe that if the update time $h$ is driven to zero, then $\Delta=0$. It is apparent that the results are influenced by the choice of $K$.

\section{An Output Feedback Networked Control System}

We now extend our approach to include plants where the state is not directly measurable. In this case, in order to obtain an estimate of the plant state vector, a state observer is used. It is assumed that the state observer is collocated with the sensor. Again, we use the plant model, $\dot{\hat{x}}=\hat{A} \hat{x}+\hat{B} u$, to design the state observer. See Figure 2 .

The observer has as inputs the output and input of the plant. In the implementation, in order to acquire the input of the plant, which is at the other side of the communication link, the observer can have a version of the model and controller, and knowledge of the update time $h$.

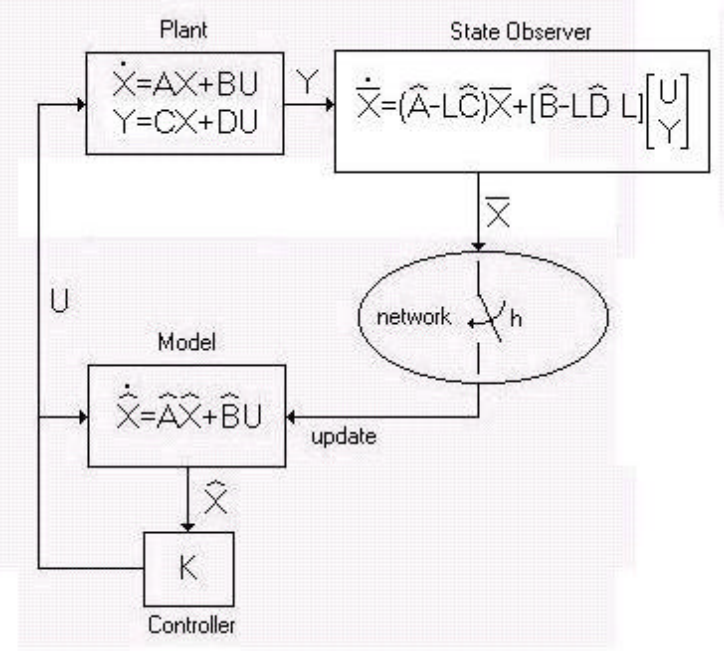

Figure 2: Proposed configuration of an output feedback networked control system.

In summary, the system dynamic equations are for $t \in\left[t_{k}, t_{k+1}\right)$ :

$$
\begin{array}{lll}
\text { Plant: } & \dot{x}=A x+B u, \quad y=C x+D u \\
\text { Model: } & \dot{\hat{x}}=\hat{A} \hat{x}+\hat{B} u, \quad y=\hat{C} \hat{x}+\hat{D} u \\
\text { Controller: } & u=K \hat{x} & \\
\text { Observer: } & \dot{\bar{x}}=(\hat{A}-L \hat{C}) \bar{x}+\left[\begin{array}{ll}
\hat{B}-L \hat{D} & L
\end{array}\right]\left[\begin{array}{l}
u \\
y
\end{array}\right] .
\end{array}
$$

We now proceed in a similar way as in the previous case of full state feedback. Namely, there will be an update interval $h$, after which the observer updates the controller's model state $\hat{x}$ with its estimate $\bar{x}$. We define an error $e$ that is the difference between the controller's model state and the observer's estimate: $e=\bar{x}-\hat{x}$. It is clear that at times $t_{k}$, where $t_{k}-t_{k-1}=h$, the error $e$ will be equal to zero. We also define the modeling error matrices in the same way as before: $\tilde{A}=A-\hat{A}, \quad \tilde{B}=B-\hat{B}, \tilde{C}=C-\hat{C}$, $\tilde{D}=D-\hat{D}$.

Now for $t \in\left[t_{k}, t_{k+1}\right)$, we have $u=K \hat{x}$ and:

$$
\begin{aligned}
& \dot{x}=A x+B K \hat{x} \\
& \dot{\hat{x}}=(\hat{A}+\hat{B} K) \hat{x} \\
& \dot{\bar{x}}=\left[\begin{array}{lll}
L C & \hat{B} K+L \tilde{D} K & \hat{A}-L \hat{C}
\end{array}\right]\left[\begin{array}{lll}
x & \hat{x} & \bar{x}
\end{array}\right]^{T}
\end{aligned}
$$

with initial conditions $\hat{x}\left(t_{k}\right)=\bar{x}\left(t_{k}\right)$. Then the dynamics of the overall system for $t \in\left[t_{k}, t_{k+1}\right)$ can be described by:

$$
\dot{z}=\Lambda_{o} z \text { for } t \in\left[t_{k}, t_{k+1}\right), z\left(t_{k}\right)=\left[\begin{array}{lll}
x\left(t_{k}^{-}\right) & \bar{x}\left(t_{k}^{-}\right) & 0
\end{array}\right]^{T}
$$

where $z=\left[\begin{array}{lll}x & \bar{x} & e\end{array}\right]^{T}$, and: 


$$
\Lambda_{o}=\left[\begin{array}{ccc}
A & B K & -B K \\
L C & \hat{A}-L \hat{C}+\hat{B} K+L \tilde{D} K & -\hat{B} K-L \tilde{D} K \\
L C & L \tilde{D} K-L \hat{C} & \hat{A}-L \tilde{D} K
\end{array}\right]
$$

\section{Proposition \#2}

The system with dynamics described by (7) with initial conditions $z\left(t_{0}\right)=\left[\begin{array}{lll}x\left(t_{0}\right) & \bar{x}\left(t_{0}\right) & 0\end{array}\right]^{T}=z_{0}, \quad t_{0}=0$, has the following response:

$$
z(t)=e^{\Lambda_{o}\left(t-t_{k}\right)}\left(\left[\begin{array}{lll}
I & 0 & 0 \\
0 & I & 0 \\
0 & 0 & 0
\end{array}\right] e^{\Lambda h}\left[\begin{array}{ccc}
I & 0 & 0 \\
0 & I & 0 \\
0 & 0 & 0
\end{array}\right]\right)^{k} z_{0},
$$

for $t \in\left[t_{k}, t_{k+1}\right)$, with $\quad t_{k+1}-t_{k}=h$

In the following, we present the necessary and sufficient conditions for this system to be exponentially stable at large (or globally).

\section{Theorem \#2}

The system described by (7) is globally exponentially stable around the solution $z=\left[\begin{array}{lll}x & \bar{x} & e\end{array}\right]^{T}=\left[\begin{array}{lll}0 & 0 & 0\end{array}\right]^{T}$ if and only if the eigenvalues of $M_{o}=\left[\begin{array}{lll}I & 0 & 0 \\ 0 & I & 0 \\ 0 & 0 & 0\end{array}\right] e^{\Lambda_{o} h}\left[\begin{array}{lll}I & 0 & 0 \\ 0 & I & 0 \\ 0 & 0 & 0\end{array}\right]$ are inside the unit circle.

The proof for this theorem is analogous to the one for Theorem 1 and can be found in the technical report (Montestruque \& Antsaklis, 2002).

The eigenvalues of the test matrix $M_{o}$ can be studied in a similar fashion to those of the state feedback case. By replacing $h$ by $t$, applying the Laplace transform and isolating the nonzero upper left block of $M_{o}$ we obtain:

$$
P=\left[\begin{array}{cc}
(s I-\hat{A}-\hat{B} K)^{-1}+\Delta_{1} & (s I-A)^{-1} B K(s I-\hat{A}-\hat{B} K)^{-1} \\
\Delta_{3} & (s I-\hat{A}+L \hat{C})^{-1}+\Delta_{2}
\end{array}\right]
$$

$\Delta_{1}=(s I-A)^{-1}(\tilde{A}+\tilde{B} K)(s I-\hat{A}-\hat{B} K)^{-1}$

$$
\Delta_{2}=(s I-\hat{A}+L \hat{C})^{-1} \text {. }
$$

$$
\left(\tilde{B} K-(\tilde{A}-L \tilde{C})(s I-A)^{-1} B K\right)(s I-\hat{A}-\hat{B} K)^{-1}
$$

$\Delta_{3}=(s I-\hat{A}+L \hat{C})^{-1}$.

$$
\begin{aligned}
& \left(\tilde{A}-L \tilde{C}+\tilde{B} K-(\tilde{A}-L \tilde{C})(s I-A)^{-1}(\tilde{A}+\tilde{B} K)\right) \\
& (s I-\hat{A}-\hat{B} K)^{-1}
\end{aligned}
$$

It is clear that if $\tilde{A} \rightarrow 0, \quad \tilde{B} \rightarrow 0$, and $\tilde{C} \rightarrow 0$ then $\Delta_{1} \rightarrow 0, \quad \Delta_{2} \rightarrow 0, \quad$ and $\Delta_{3} \rightarrow 0$ from here it can be seen that by making the error between the plant and the model zero, we obtain the classic state feedback-observer architecture. From the structure of matrix $P$ it is clear that the separation principle does not apply when the model-plant error is not zero.

\section{The Discrete Time Domain}

We now extend our results to discrete time plants. We will assume that the update interval $h$ will be an integer, representing the number of clock tics after which the actuator's model is updated.

Consider the following equations:

Plant: $\quad x(n+1)=A x(n)+B u(n), y(n)=C x(n)+D u(n)$

Model: $\hat{x}(n+1)=\hat{A} \hat{x}(n)+\hat{B} u(n), y(n)=\hat{C} \hat{x}(n)+\hat{D} u(n)$

Controller: $u(n)=K \hat{x}(n)$

Observer: $\bar{x}(n+1)=(\hat{A}-L \hat{C}) \bar{x}(n)+\left[\begin{array}{ll}\hat{B}-L \hat{D} & L\end{array}\right]\left[\begin{array}{l}u(n) \\ y(n)\end{array}\right]$

$$
\text { for } n \in\left[n_{k}, n_{k+1}\right), \quad \text { with } \quad n_{k+1}-n_{k}=h
$$

Below the results for the full state feedback case are presented. Proofs will be omitted and can be found in the report (Montestruque \& Antsaklis, 2002).

\section{Theorem \#3}

The discrete state feedback system is globally exponentially stable around the solution $z=\left[\begin{array}{ll}x & e\end{array}\right]^{T}=\left[\begin{array}{ll}0 & 0\end{array}\right]^{T}$ if and only if the eigenvalues of $M_{D}=\left[\begin{array}{ll}I & 0 \\ 0 & 0\end{array}\right] \Lambda_{D}{ }^{h}\left[\begin{array}{ll}I & 0 \\ 0 & 0\end{array}\right]$ are inside the unit circle, where $\Lambda_{D}=\left[\begin{array}{cc}A+B K & -B K \\ \tilde{A}+\tilde{B} K & \hat{A}-\tilde{B} K\end{array}\right]$.

\section{Theorem \#4}

The discrete output feedback system is globally exponentially stable around the solution $z=\left[\begin{array}{lll}x & \bar{x} & e\end{array}\right]^{T}=\left[\begin{array}{lll}0 & 0 & 0\end{array}\right]^{T}$ if and only if the eigenvalues of $M_{F}=\left[\begin{array}{lll}I & 0 & 0 \\ 0 & I & 0 \\ 0 & 0 & 0\end{array}\right] \Lambda_{F}{ }^{h}\left[\begin{array}{lll}I & 0 & 0 \\ 0 & I & 0 \\ 0 & 0 & 0\end{array}\right]$ are inside the unit circle,

$$
\Lambda_{F}=\left[\begin{array}{ccc}
A & B K & -B K \\
L C & \hat{A}-L \hat{C}+\hat{B} K+L \tilde{D} K & -\hat{B} K-L \tilde{D} K \\
L C & L \tilde{D} K-L \hat{C} & \hat{A}-L \tilde{D} K
\end{array}\right] .
$$
where

\section{A State Feedback Networked Control System in a}

\section{Network with Communication Delays}

Previously we assumed that the network delays were negligible. This is usually true for plants with slow dynamics relative to the network bandwidth. Next we extend our results to include the case where transmission delay is present. We assume that the update time $h$ is larger than the 
delay time $\tau$, and that the update time $h$ and the delay $\tau$ are constant.

At times $k h$ - $\tau$ the sensor transmits the state data to the controller/actuator. This data will arrive $\tau$ seconds latter. So, at times $k h$ the controller/actuator receives the state vector value $x(k h-\tau)$. The main idea is to use the plant model in the controller/actuator to calculate the present value of the state. The state approximate obtained can be used to update the controller's model as in previous setups. The system is depicted in Figure 3.

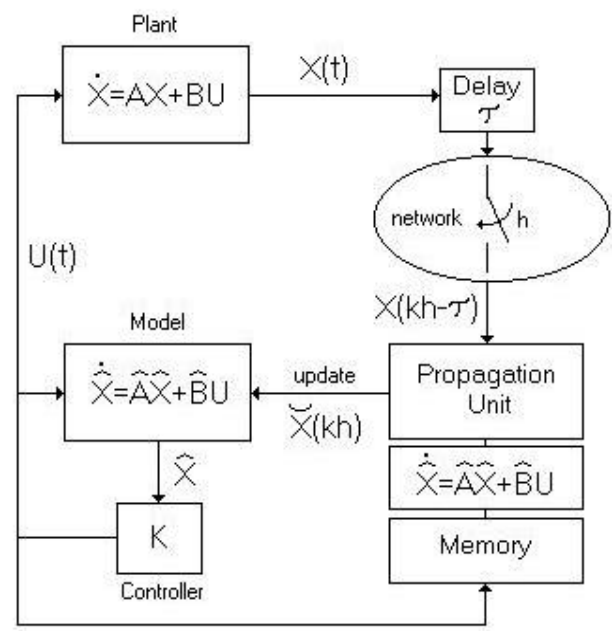

Figure 3: Proposed configuration of a state feedback networked control system in the presence of network delays.

The Propagation Unit uses the plant model and the past values of the control input $u(t)$ to calculate an estimate of actual state $\breve{x}(k h)$ from the received data $x(k h-\tau)$. This estimate is then used to update the model that with the controller will generate the control signal for the plant.

The system is then described by the following equations:

$$
\begin{array}{ll}
\text { Plant: } & \dot{x}=A x+B u \\
\text { Model: } & \dot{\hat{x}}=\hat{A} \hat{x}+\hat{B} u \\
\text { Controller: } & u=K \hat{x}, \quad t \in\left[t_{k}, t_{k+1}\right) \\
\text { Propagation Unit: } \dot{\vec{x}}=\hat{A} \breve{x}+\hat{B} u, \quad t \in\left[t_{k+1}-\tau, t_{k+1}\right] & \\
\text { Update law: } & \left\{\begin{array}{l}
\vec{x} \leftarrow x, t=t_{k+1}-\tau \\
\hat{x} \leftarrow \breve{x}, t=t_{k+1}
\end{array}\right\}
\end{array}
$$

To simplify the analysis, we initialize the propagation unit at time $t_{k+1}-\tau$ with the state vector that the sensor transmitts. We then run the plant, model, and propagation unit together until $t_{k+1}$. At this time, the model is updated with the propagation unit state vector, as described in the update law of (8). This is equivalent to having the propagation unit receive the state vector $x\left(t_{k+1}-\tau\right)$ at $t_{k+1}$ and propagating it instantaneously to $t_{k+1}$.

We define the errors $\hat{e}=\breve{x}-\hat{x}$ and $\breve{e}=x-\breve{x}$. We also make the following definitions:

$$
\begin{aligned}
\Lambda_{T} & =\left[\begin{array}{ccc}
A+B K & -B K & -B K \\
\tilde{A}+\tilde{B} K & \hat{A}-\tilde{B} K & -\tilde{B} K \\
0 & 0 & \hat{A}
\end{array}\right], z=\left[\begin{array}{c}
x \\
\breve{e} \\
\hat{e}
\end{array}\right] \\
I_{T 1} & =\left[\begin{array}{lll}
I & 0 & 0 \\
0 & I & 0 \\
0 & 0 & 0
\end{array}\right], I_{T 2}=\left[\begin{array}{ccc}
I & 0 & 0 \\
0 & 0 & 0 \\
0 & I & 0
\end{array}\right]
\end{aligned}
$$

With these definitions we proceed to present the system described by (8) in a compact form. The dynamics of the overall system for $t \in\left[t_{k}, t_{k+1}\right)$ can be described by

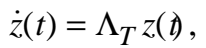

with state reset equations: $z\left(t_{k}\right)=\left[\begin{array}{lll}x\left(t_{k}^{-}\right) & \breve{e}\left(t_{k}^{-}\right) & 0\end{array}\right]^{T}$,

$$
\text { and } z\left(t_{k+1}-\tau\right)=\left[\begin{array}{c}
x\left(\left(t_{k+1}-\tau\right)^{-}\right) \\
0 \\
\breve{e}\left(\left(t_{k+1}-\tau\right)^{-}\right)+\hat{e}\left(\left(t_{k+1}-\tau\right)^{-}\right)
\end{array}\right]
$$

and with $t_{k+1}-t_{k}=h, \quad 0<\tau<h$.

\section{Proposition \#3}

The system with dynamics described by (9) with initial conditions $z\left(t_{0}\right)=\left[\begin{array}{lll}x_{0} & \breve{e}_{0} & \hat{e}_{0}\end{array}\right]^{T}=z_{0}, t_{0}=0$, has the following response:

$$
\begin{gathered}
z(t)=e^{\Lambda_{T}\left(t-t_{k}\right)}\left(I_{T 1} e^{\Lambda_{T} \tau} I_{T 2} e^{\Lambda_{T}(h-\tau)}\right)^{k}\left[\begin{array}{lll}
x_{0} & \breve{e}_{0} & \hat{e}_{0}
\end{array}\right]^{T} \\
\text { for } t \in\left[t_{k}, t_{k+1}-\tau\right) \\
z(t)=e^{\Lambda_{T}\left(t-t_{k+1}+\tau\right)} I_{T 2} e^{\Lambda_{T}(h-\tau)} \cdot \\
\left(I_{T 1} e^{\Lambda_{T} \tau} I_{T 2} e^{\Lambda_{T}(h-\tau)}\right)^{k}\left[\begin{array}{lll}
x_{0} & \breve{e}_{0} & \hat{e}_{0}
\end{array}\right]^{T}
\end{gathered}
$$

for $t \in\left[t_{k+1}-\tau, t_{k+1}\right)$.

In the following the necessary and sufficient conditions for this system to be exponentially stable at large (or globally) are presented.

\section{Theorem \#3}

The system described by (9) is globally exponentially stable around the solution $z=\left[\begin{array}{lll}x & \breve{e} & \hat{e}\end{array}\right]^{T}=\left[\begin{array}{lll}0 & 0 & 0\end{array}\right]^{T}$ if and only if the eigenvalues of $M_{T}=\left[\begin{array}{lll}I & 0 & 0 \\ 0 & I & 0 \\ 0 & 0 & 0\end{array}\right] e^{\Lambda_{T} \tau}\left[\begin{array}{lll}I & 0 & 0 \\ 0 & 0 & 0 \\ 0 & I & I\end{array}\right] e^{\Lambda_{T}(h-\tau)}$ are inside the unit circle.

It is interesting to note that the results on Theorem \#3 can be seen as a generalization of Theorem \#1. This can be shown by driving $\tau$ to zero. The details for the proof of Proposition \#3 and Theorem \#3 can be found in the technical report (Montestruque \& Antsaklis, 2002). 


\section{Conclusions}

The control architecture introduced in this paper represents a natural way of placing critical information about the plant on the network so to reduce the data traffic load. By making the sensor and actuator more "intelligent" the networked control system is able to predict the future behavior of the plant, and send the precise information at critical times so to ensure plant stability.

Performance of the control system is also important and is currently under investigation. Note that the techniques used here are related to the ones known as lifting operators (Bamieh, Pearson, Francis, \& Tannenbaum, 1991; Mirkin et al., 1999), where it has been shown that plant induced norms are invariant under these lifting operators.

Note that the some of the results presented here have appeared earlier in the conference proceedings Montestruque \& Antsaklis (2002).

\section{Acknowledgements}

The partial support of the National Science Foundation (NSF ECS99-12458 \& CCR01-13131), and of the DARPA/ITO-NEST Program (AF-F30602-01-2-0526) is gratefully acknowledged.

\section{References}

Bamieh, B., Pearson, J.B., Francis, B., \& Tannenbaum, A. (1991). A Lifting Technique For Linear Periodic Systems With Applications To Sampled-Data Control. Systems \& Control Letters, Vol 12, pp.79-88.

Brockett, R. (1997). Minimum Attention Control. Proceedings of the 36th Conference on Decision and Control, pp. 2628-2632.

Elia, N., \& Mitter, S. (2001). Stabilization of Linear Systems With Limited Information. IEEE Transactions on Automatic Control, pp. 1384-1400.

Hristu-Varsakelis, D. (2001). Feedback Control Systems as Users of a Shared Network: Communication Sequences that Guarantee Stability. Proceedings of the $40^{\text {th }}$ IEEE Conference on Decision and Control, pp. 3631-3636.

Ishii, H., \& Francis, B. (2000). Stabilization With Control Networks. Control 2000, Cambridge UK.

Matveev, A.S., \& Savkin, A.V. (2001). Optimal Control of Networked Systems via Asynchronous Communication Channels with Irregular Delays. Proceedings of the $40^{\text {th }}$ IEEE Conference on Decision and Control, pp. 2323-2332.

Mirkin, L., Rotstein, H., \& Palmor, H. (1999). $\mathrm{H}_{2}$ and $\mathrm{H}_{\mathrm{inf}}$ Design Of Sampled-Data Systems Using Lifting. Part I: General Framework And Solutions. SIAM Journal Of Control And Optimization, Vol 38, No. 1, pp. 175-196.

Montestruque, L.A., \& Antsaklis, P. (2002). Model-Based Networked Control Systems: Stability. ISIS Technical Report ISIS-2002-001. University of Notre Dame, www.nd.edu/ isis/.

Montestruque, L.A., \& Antsaklis, P. (2002). Model-Based Networked Control Systems: Necessary and Sufficient Conditions for Stability. $10^{\text {th }}$ Mediterranean Conference on Control and Automation.

Montestruque, L.A., \& Antsaklis, P. (2002). State and Output Feedback Control in Model-Based Networked Control Sy stems. $41^{\text {st }}$ IEEE Conference on Decision and Control.

Nair G., \& Evans, R. (2000). Communication-Limited Stabilization of Linear Systems. Proceedings of the Conference on Decision and Control, pp. 1005-1010.

Petersen, I.R., \& Savkin, A.V. (2001). Multi-rate Stabilization of Multivariable Discrete-Time Linear Systems via a Limited
Capacity Communication Channel. Proceedings of the $40^{\text {th }}$ IEEE Conference on Decision and Control, pp. 304-309.

Rehbinder, H., \& Sanfridson, M. (2000). Scheduling Of A Limited Communication Channel For Optimal Control. Proceedings of the Conference on Decision and Control, pp. 10111016.

Walsh, G., Beldiman, O., \& Bushnell, L. (1999). Asymptotic Behavior of Networked Control Systems. Proceedings of the International Conference on Control Applications.

\section{Appendix A. Example of a State Feedback Networked Control System.}

An example is given on how the theorem can be applied if two entries on the $A$ matrix of the model can vary within a certain interval.

$$
\begin{array}{ll}
\text { model: } & \hat{A}=\left[\begin{array}{ll}
0 & 1 \\
0 & 0
\end{array}\right], \hat{B}=\left[\begin{array}{l}
0 \\
1
\end{array}\right] ; \\
\text { plant: } & A=\left[\begin{array}{cc}
0 & 1+\tilde{a}_{12} \\
0+\tilde{a}_{21} & 0
\end{array}\right], B=\left[\begin{array}{l}
0 \\
1
\end{array}\right] ; \\
& \text { with } \tilde{a}_{12}=[-0.5,0.5], \tilde{a}_{21}=[-0.5,0.5]
\end{array}
$$

controller: $\quad K=[-1,-2]$.

Figure 4 shows the contour levels representing the maximum eigenvalue magnitude for the test matrix $M$ as a function of the values of the entries $(1,2)$ and $(2,1)$ of the plant matrix $A$. The update time $h$ was 2.5 seconds

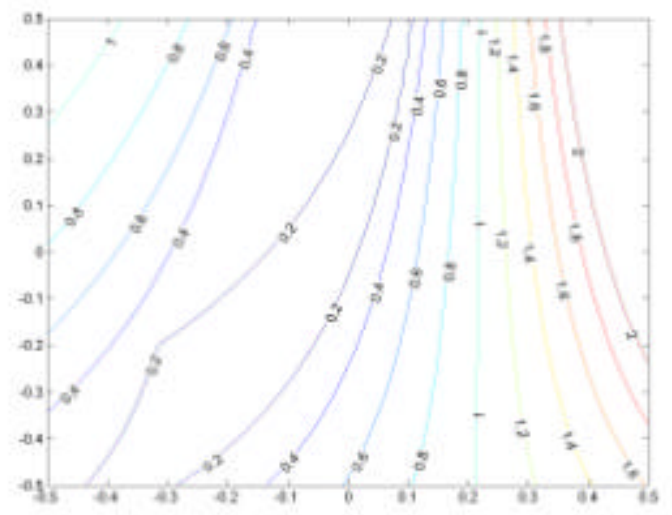

Figure 4: Maximum Eigenvalue Magnitude vs Model Error

It is easy to isolate the stable and unstable regions in the uncertainty parameter plane. The stable region lies in between the two contours labeled one. 